\title{
The Leidenfrost transition of water droplets impinging onto a superheated surface
}

\author{
Ophélie Caballina $^{* 1}$, Romain Collignon ${ }^{1}$, Abhishek Srivastava ${ }^{1}$, Fabrice Lemoine ${ }^{1}$, Guillaume \\ Castanet $^{1}$ \\ ${ }^{1}$ Université de Lorraine, CNRS, LEMTA, F-54000 Nancy, France \\ ${ }^{*}$ Corresponding author email: ophelie.caballina@univ-lorraine.fr
}

\begin{abstract}
Heat transfer during the impact of a droplet on a sapphire substrate is investigated by means of infrared thermography. For that, the sapphire is coated with a very thin layer of TiAIN having a high emissivity in the IR domain. Spatially and time resolved measurements of the temperature at the front face of the solid wall are obtained. Results obtained for water droplets show that the dynamic Leidenfrost point (LFP) is close to $450^{\circ} \mathrm{C}$ and coincides with the onset of a fingering pattern. Approaching the dynamic LFP, despite the cooling by the droplet, the wall surface temperature never decreases below $310^{\circ} \mathrm{C}$ which is about the temperature of the spinodal for water, i.e. the maximum temperature at which water can still exist in the liquid state. Considering that a wetting contact is taking place below the dynamic LFP, wall temperature measurements demonstrate that the drop impact comes with a very strong superheating of the liquid. The liquid touching the wall is heated up to the spinodal temperature. Based on the idea that the dynamic LFP could correspond to the wall temperature, for which the contact temperature at a solid/liquid interface is equal to the spinodal temperature, a model is proposed for the dynamic LFP. This model considers the thermal effusivities of the liquid and the wall, as well as the liquid flow in the spreading lamella.
\end{abstract}

\section{Keywords}

Leidenfrost temperature, Transition boiling, Film boiling, Infrared thermography

\section{Introduction}

The impact of liquid droplets on overheated solid surfaces is a phenomenon that is found in many industrial applications, such as the quenching of metal surfaces in the steel industry, loss-of-coolant accident (LOCA) in nuclear facilities, direct injection in diesel and gasoline engines where fuel droplets collide with the overheated surface of the piston. In many technological processes, spray cooling is used when it is required to obtain a rapid and efficient cooling of hot surfaces. However, one of the main challenges is to control the heat flux from the solid surface through the boiling process. Numerous studies have been motivated to obtain quantitative information, but it is still difficult to obtain the physical understanding of the boiling process during the drop impact. A fairly comprehensive review of the phenomena associated with the interaction between a droplet and a very hot surface can be found in review papers such as Liang and Mudawar [1]. Because of the different phenomena associated with the boiling (namely drop evaporation, Marangoni related effects, nucleate boiling, transition boiling, film boiling), the heat flux is not a monotonous function of the surface temperature. It is widely considered that its minimum value occurs at the so-called Leidenfrost point (LFP) which separates two major boiling regimes: the transition boiling regime and the film boiling regime. In the boiling film, the droplet sits on a vapor cushion that prevents a direct contact with the solid surface. The very low thermal conductivity of the vapor means that heat transfer is quite low in this regime. In the transition boiling regime, liquid contact occurs with portions of the surface, resulting in a significant improvement in heat transfer compared to film boiling. To predict the LFP, several models and correlations have been proposed [2-3]. These are based in several hypotheses including a Taylor-like hydrodynamic instability that can disrupt 


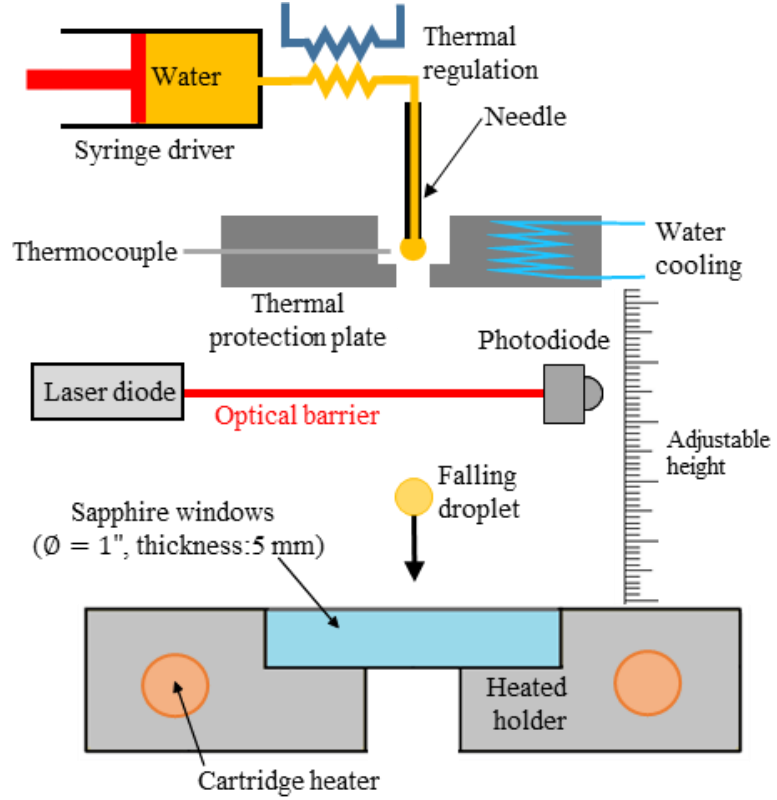

Figure 1. Experimental set-up.

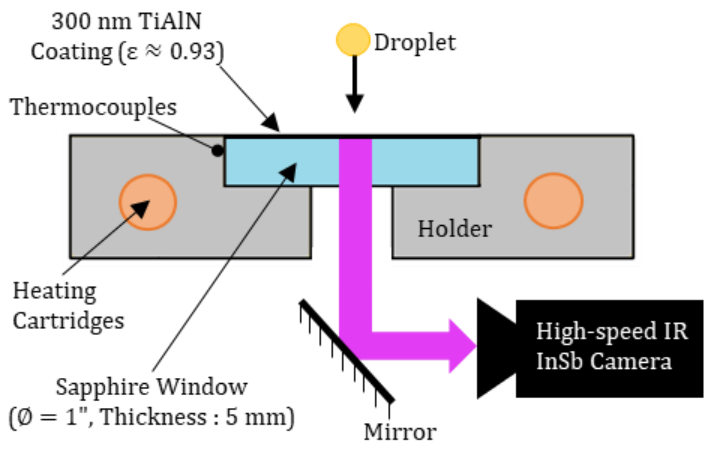

Figure 2. Optical setup used for the visualization of the temperature field at the impact surface by IR thermography.

vapor pockets, or an explosive boiling caused by homogeneous nucleation. The maximum attainable temperature to which a liquid can be heated before it vaporizes spontaneously, can be determined theoretically based on thermomechanical stability from the equation of state such as Van der Waals (the spinodal limit in the domain of metastable states is $T_{\text {spin }}=$ $320.25^{\circ} \mathrm{C}$ for pure water at one bar, as calculated with the Wagner and Pruß equation of state [4]) or from the kinetic homogeneous nucleation theory (molecular fluctuations occur in such a way to cause a localized decrease in the liquid density, leading to the formation of vapor embryos) [2,5]. Therefore, a physical contact between the liquid phase and the solid surface is only possible if the cooling is strong enough to have the surface temperature lower than this limit. However, to many authors [2, 6], it is doubtful that a model for homogeneous nucleation could explain all the experimental observations for the Leidenfrost transition.

Recently, Khavari et al.[7] developed an optical method based on total internal reflection (TIR). The latter allows revealing the formation of a fingering pattern in the boiling transition regime when applied to impinging ethanol droplets. The fingers can be easily distinguished from white spots and pockets observed at lower surface temperature in the bubbly boiling regime. Khavari et al. [7] also pointed out that the Leidenfrost transition is not an abrupt change between the 'contact-boiling' regime characterized by a violent bubbling, and the film boiling regime where the droplet never physically touches the solid surface. Their results indicates that the wetting area decreases continuously as the wall temperature approaches the LFP. Since heat transfer is mostly through the wetting surface area, a reduction of the wetting contact area seems congruent with the general idea of a heat transfer reduction when approaching the LFP. However, direct measurements of the heat transfer would supplement these observations as they will provide quantitative information on the surface temperature and local heat flux, which are key parameters to describe the boiling. Chaze et al. [8] demonstrated that it is possible to use IR thermography to reconstruct the time and space distribution of the wall heat flux during the impact of a droplet onto a superheated surface. In the present study, the above-mentioned measurement technique (IR thermography) is used to characterize the heat transfer in the Leidenfrost transition for an impinging water droplet.

\section{Experimental set-up and measurement techniques}

Experimental set-up 
A syringe is used to produce water drops with a diameter $d_{0}$ of $2.6 \mathrm{~mm}$ (Fig. 1). After detachment from the needle of the syringe, the falling water droplets impinges on a sapphire window (25.4 $\mathrm{mm}$ in diameter and $5 \mathrm{~mm}$ thick) placed on a steel holder which is heated by cartridge heaters. In the present study, the initial temperature of the sapphire $T_{w 0}$ is changed between $300^{\circ} \mathrm{C}$ and $700^{\circ} \mathrm{C}$. The impact velocity of the droplet $U_{0}$ is adjusted by changing the needles height. In the following, the Weber number $W e=\rho U_{0}^{2} d_{0} / \gamma$ ranges between 17 and 140 ( $\gamma$ stands for the surface tension). A water-cooled protective plate is required to prevent the liquid from heating up inside the needle used to produce the droplet.

Temperature measurement and heat flux reconstruction at the solid surface using IR thermography

The temperature of the impact surface is characterized by means of an IR camera (FLIR ORION SC7000), which incorporates a cooled InSb detector operating in the 1.5 to $5.5 \mu \mathrm{m}$ infrared waveband. The IR camera is equipped with a high magnification objective allowing a field of view of about $7 \mathrm{~mm}$. The beginning of the acquisition by the camera is triggered by the passage of the droplet across an optical barrier (see Figure 1).

Bottom view images of the impacted surface are recorded thanks to the transparency of the sapphire substrate in the IR domain below $5 \mu \mathrm{m}$ (see Figure 2). The top face of the sapphire window, where the impacts take place, is coated with a nanolayer of TiAIN (300 nm in thickness) which is resistant to high temperature. TiAIN has a high emissivity in the detection band of the camera $(\varepsilon \approx 0.93)$. A benefit of this high emissivity is that the radiative emission from the surface is sufficiently large to have integration times of a few tens of $\mu$ s and a very limited contributions of ambient radiations (especially those coming from the heated holder). The small thickness of the TiAIN coating allows considering that the temperature measured by IR is the temperature at the upper surface of the sapphire $T_{S}$.

The distribution of the local heat flux at the wall surface $q_{w}$ can be reconstructed from the IR images using the method proposed by Chaze et al. [8]. Considering an axisymmetric heat conduction problem in the sapphire, the transient heat transfer equation in the cylindrical coordinates can be rewritten using transformations of Hankel in space and

Laplace in time. An inverse heat conduction problem can be solved using the so-called quadrupole method [9] in order to obtain an analytical relationship between the measured temperature $T_{S}$ and the heat flux $q_{W}$ at the upper surface of the solid wall, where the drop impact is taking place. A window of $160 \times 128$ pixels, allowing for a frame rate at $1.25 \mathrm{kfps}$, is used to estimate the wall heat flux $q_{w}$

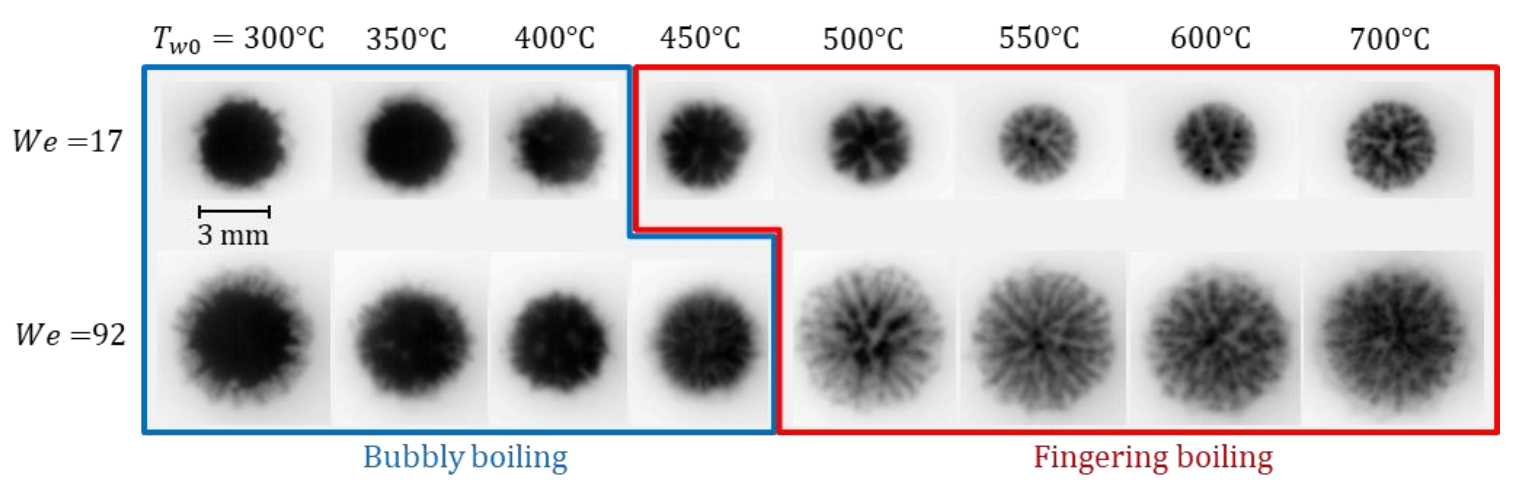

Figure 3. IR images illustrating the change of the thermal footprint of the droplet in the Leidenfrost transition. These images correspond to a time of about $3 \mathrm{~ms}$ after the beginning of the drop impact and $d_{0}=2.6 \mathrm{~mm}$. 


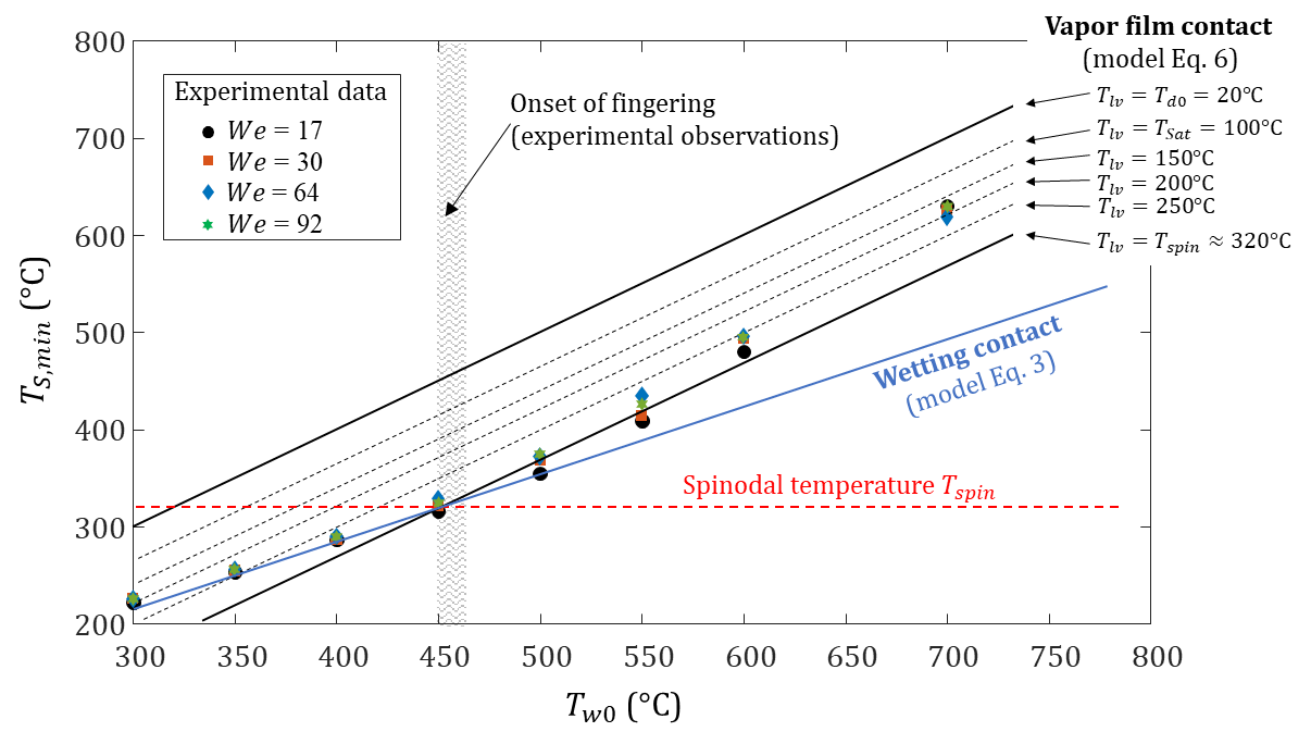

Figure 4. Maximal decrease in temperature of the solid surface during the impact process for different We.

\section{Results and Discussion}

\section{Boiling regimes}

Depending on the initial wall temperature $T_{w 0}$, two types of pattern can be evidenced on the IR images as illustrated in Figure 3. There are strong similarities with the TIR images presented by Khavari et al.[7] in the case of ethanol droplets. For $T_{W 0}>450^{\circ} \mathrm{C}$, a fingering pattern can be clearly observed. The boiling regime below $450^{\circ} \mathrm{C}$ corresponds to the bubbly boiling regime. In addition to the formation of fingers, the transition between the two boiling regimes is marked by a change in the area of effective heat transfer, especially for $W e=92$. The impingement of a water droplet on a superheated sapphire substrate has been already studied by Tran et al. [10], who examined the Leidenfrost transition based on sideview images of the impact. They located the onset of film boiling at about $450^{\circ} \mathrm{C}$ for $W e$ in the order of a few tens. The observation of Tran et al. [10] are congruent with the present results. Fingers can be observed at $T_{W 0}=450^{\circ} \mathrm{C}$ for $W e=17$, but a slight increase of the wall temperature is required for their formation at higher $W e$. When a drop impinges on the hot substrate, the surface temperature of the substrate $T_{S}$ first decreases. It reaches a minimum $T_{S, \min }$, then it eventually returns to the initial wall temperature $T_{W 0}$. Figure 4 shows the variation of $T_{S, \min }$ as a function of $T_{W 0}$. As expected, $T_{S, \min }$ never goes below the spinodal temperature $T_{\text {spin }} \approx$ $320^{\circ} \mathrm{C}$ in the film boiling regime (ie. for $T_{W 0}>450^{\circ} \mathrm{C}$ ). The LFP corresponds to an initial wall temperature $T_{W 0} \approx 450^{\circ} \mathrm{C}$, for which it is observed that $T_{S, \min }$ is about equal to $T_{\text {spin }}$. This means that it is the highest conceivable value of the LFP that is actually achieved in these drop impacts.

\section{Theoretical description of the thermal contact}

When two semi-infinite bodies initially at temperature $e_{1}$ and $T_{2}$ are brought in perfect thermal contact, the temperature at the contact surface $T_{C}$ instantly takes a value that is determined by their respective thermal effusivities $e_{1}$ and $e_{2}: T_{C}=\left(e_{1} T_{1}+e_{2} T_{2}\right) /\left(e_{1}+e_{2}\right)$. This expression for the contact temperature is valid at any time in the case of two semi-infinite bodies. This formulation for $T_{C}$ which assumes that heat transfer is only of conducive nature, is also a good approximation for the contact temperature of finite bodies. In that case, it is valid near the center of the contact region, as long as the thermal diffusion length remains small in 
comparison to the thickness of the bodies and to the dimensions of the contact area. It implicitly assumes that the heat transfer is purely by heat conduction in the two bodies. Therefore, this equation does not directly applied to the case of a spreading drop, where the internal liquid flow intensifies the heat transfer by convection and reduces the thickness of the thermal boundary layer developing in the liquid lamella $[17,26]$. As detailed by Breitenbach et al. [11], the temperature field resulting from the contact with a spreading drop can be approximately described by solving a one-dimensional heat equation in the liquid and solid phases separately. From the mentioned approach, the heat flux at the contact surface, can be determined by:

$$
\begin{aligned}
& q_{l}(t)=\frac{\sqrt{5} e_{l}\left(T_{C}-T_{d 0}\right)}{\sqrt{\pi t}} \\
& q_{w}(t)=\frac{e_{w}\left(T_{w 0}-T_{C}\right)}{\sqrt{\pi t}}
\end{aligned}
$$

where $T_{d 0}$ and $T_{w 0}$ are respectively the temperatures of the droplet and the solid substrate prior to the contact. The factor $\sqrt{5}$ in Equation (1) takes into account the enhancement of heat transfer induced by convection in the spreading lamella [11-12].

\section{Thermal contact in the partially wetting boiling regime}

When there is a wetting contact between the droplet and the solid surface, the surface temperature rapidly reaches a minimum value $T_{S, \min }$ at the center of the impact region. The temperature at this location remains about constant for a few ms. Eventually, it rises and returns to $T_{W 0}$ due to the fact that the droplet is not a semi-infinite body. Neglecting the contribution of liquid vaporization to the cooling [12], the temperature of the wetting contact can be obtained by equating the expressions of $q_{l}$ and $q_{w}$ in Eqs.(1) and (2),

$$
T_{C}=\frac{\sqrt{5} e_{l} T_{d 0}+e_{w} T_{w 0}}{\sqrt{5} e_{l}+e_{w}}
$$

As $T_{W 0}$ increases, the liquid in contact with the solid substrate is more and more superheated. The above expression of $T_{C}$ is plotted as a blue line in Figure 4. It perfectly matches the experimental data for the minimum temperature of the surface $T_{S, \min }$, until $T_{W 0}$ reaches the LFP.

\section{Leidenfrost point}

Basically, no wetting is possible if the temperature of the solid surface remains above the spinodal temperature $T_{\text {spin }}$ during the cooling process. In the scenario considered below, the rapidity of the contact is such, that the liquid temperature reaches the maximum conceivable value (i.e. the spinodal point $T_{\text {spin }}$ ). This implies a sufficiently large impact velocity, since the dynamics of the lubrication layer of gas are extremely important at low velocities (Even a drop deposited on an unheated surface can glide on a trapped air cushion at low impact velocities [13]). Assuming $T_{S, \min }$ is equal to $T_{\text {spin }}$ in the followings, it is possible to obtain an expression for the dynamic LFP. Using Eq.(3), we can write:

$$
T_{C}=T_{\text {spin }}=\frac{\sqrt{5} e_{l} T_{d 0}+e_{w} L F P}{\sqrt{5} e_{l}+e_{w}}
$$

From this expression, the dynamic LFP can be determined by: 


$$
L F P=T_{\text {spin }}+f(W e, R e) \cdot \frac{e_{l}}{e_{w}} \cdot\left(T_{\text {spin }}-T_{d 0}\right)
$$

where $f=\sqrt{5}$ for the non-viscous velocity field. Considering $\kappa_{w}=14 \mathrm{~W} \cdot \mathrm{m}^{-1} \cdot \mathrm{K}^{-1}, C_{p w}=$ $1150 \mathrm{~J} \cdot \mathrm{kg}^{-1} \cdot \mathrm{K}^{-1}$ and $\rho_{w}=3980 \mathrm{~kg} \cdot \mathrm{m}^{-3}$ for the thermal properties of sapphire, $e_{w}$ is about $8000 \mathrm{~J} \cdot \mathrm{K}^{-1} \cdot \mathrm{m}^{-2} \cdot \mathrm{s}^{-1 / 2}$. For water, $\kappa_{l}=0.6 \mathrm{~W} \cdot \mathrm{m}^{-1} \cdot \mathrm{K}^{-1}, \quad C_{p l}=4180 \mathrm{~J} \cdot \mathrm{kg}^{-1} \cdot \mathrm{K}^{-1}$ and $\rho_{l}=$ $1000 \mathrm{~kg} . \mathrm{m}^{-3}$, which yields $e_{l}=1580 \mathrm{~J} \cdot \mathrm{K}^{-1} \cdot \mathrm{m}^{-2} \cdot \mathrm{s}^{-1 / 2}$. Applying Eq.(5), the LFP is about $453^{\circ} \mathrm{C}$, which is consistent with the experimental observations (evidence of the onset of the fingering boiling). According to Eq.(5), the liquid subcooling $T_{S a t}-T_{d 0}$ has a weak influence on LFP, since $T_{d 0}$ is small compared to $T_{\text {spin }}$. This trend is also in good agreement with the literature [2]. The velocity field is only valid for relatively large impact velocities, when liquid inertia is predominant over viscous and capillary forces. Typically, $W e$ has to be in the range of 10 , while $R e$ has to be of a few hundreds [14]. For the low impact velocities, it can be anticipated that $f$ takes a smaller value tending towards 1 when $U_{0}$ is approaching 0 . This corresponds to a LFP tending toward $380^{\circ} \mathrm{C}$ according to Eq.(5). This agrees well with the results of Tran et al. [10], who found a dynamic LFP close to $380^{\circ} \mathrm{C}$ for $W e=4$. Hence, Eq.(5) seems to hold for this relatively low value of $W e$.

\section{Liquid superheating}

In the film boiling regime, Eq.(1) can be modified to determine the heat flux $q_{l}$ entering into the droplet. The temperature $T_{C}$ in Eq.(1) is replaced by $T_{l v}$ the temperature at the liquid/vapor interface, which leads to $q_{l}(t)=\sqrt{5} e_{l}\left(T_{l v}-T_{d 0}\right) / \sqrt{\pi t}$.

This equation implies that the temperature of the liquid/vapor interface $T_{l v}$ remains approximately constant during the impact process. This assumption is made in almost all the models for the film boiling, which presumes that $T_{l v}=T_{S a t}$.

Equating the expressions of $q_{l}$ and $q_{w}$, and replacing $T_{C}$ by $T_{l v}$ on the liquid side, one can determine the contact temperature $T_{C}$ at the solid surface, which is expected to compare with $T_{S, \min }$ :

$$
T_{S, \min }=T_{C}=T_{w 0}-\sqrt{5} \cdot \frac{e_{l}}{e_{w}} \cdot\left(T_{l v}-T_{d 0}\right)
$$

The surface temperature $T_{S, \min }$ determined by Eq.(6) is plotted in Figure 4 as a function of $T_{w 0}$. These are parallel lines that are shifted vertically for each value of $T_{l v}$ being considered. In most of the models for the film boiling, it is assumed that $T_{l v}=T_{\text {Sat }}[11,12]$. However, with this assumption, the cooling of the solid surface is by far too small in comparison with the experiments. To have a better agreement, it is necessary to consider that the liquid/vapor interface can be largely superheated in the film boiling regime. Until $T_{w 0}=550^{\circ} \mathrm{C}, T_{l v}$ remains about equal to $T_{\text {spin }}$. Then, increasing $T_{w 0}$, the superheating of the liquid/vapor interface becomes less and less important. At $T_{w 0}=700^{\circ} \mathrm{C}, T_{l v}$ is still about $200^{\circ} \mathrm{C}$. Presumably, it would have been necessary to raise the wall temperature to a much higher value in order to have actually $T_{l v}$ equal to $T_{S a t}$. The above description suggests that the fingering boiling is in fact a form of homogeneous nucleation. At the very beginning of impact, the liquid is heated 


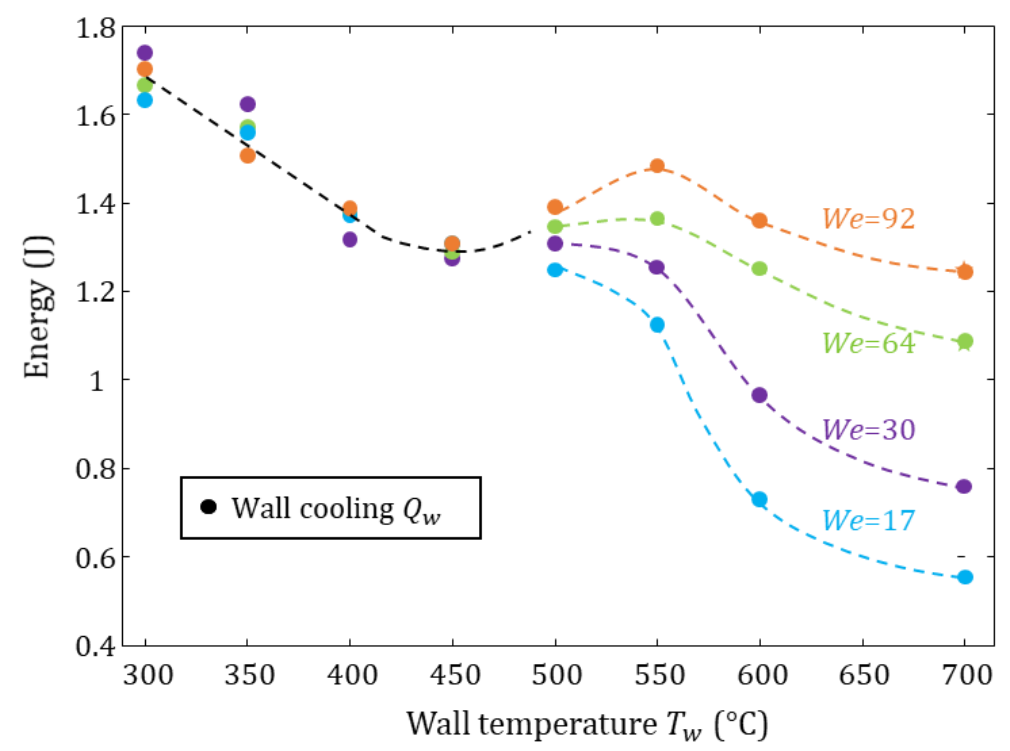

Figure 5. Experimental data showing the evolution of the heat taken from the solid surface $Q_{w}$ as a function of the wall temperature $T_{w 0}$. The smooth curves with dotted lines are introduced here to highlight trends in these experimental data.

so strongly in a small layer of liquid close to the liquid/vapor interface, that it becomes superheated. This permits the formation of numerous vapor bubbles by homogeneous nucleation. The liquid layer where homogeneous nucleation occurs, is very thin (typically just a few $\mu \mathrm{m}$ ), because its thickness must remain small in comparison to that of the thermal boundary layer. The vapor bubbles are then transported by the liquid flow. This transport is more efficient than in the case of bubbly boiling and heterogeneous nucleation. It is not restrained by the pinning of triple contact lines (bubbles attached on the solid surface). During their transport, the vapor bubbles undergo an elongation in the radial direction. The growth and elongation of the bubbles accelerates the process of bubble coalescence, which results in the formation of separated fingers. The vapor is mainly conveyed to the edge of the spreading drop by flowing into the ridges that separate the fingers.

Heat transfer in the Leidenfrost transition

To conclude, the Leidenfrost transition is highlighted by considering the heat taken to the wall $Q_{w}$. This latter can be determined by integrating the local heat flux $q_{w}$ over time and space [16]. The results are presented in Figure 5. A minimum of $Q_{w}$ is found around $T_{w 0}=450^{\circ} \mathrm{C}$ which corresponds to the LFP. However, it is not an absolute minimum, but simply a local minimum, which is weakly pronounced for the low We such as $\mathrm{We}=17$ and $\mathrm{We}=30$.

In the bubbly boiling regime (from $300^{\circ} \mathrm{C}$ to $450^{\circ} \mathrm{C}$ ), $Q_{w}$ is decreasing with $T_{w 0}$. This is mainly because an increasing fraction of the lamella at the periphery of the drop is levitated over a vapor film, which acts as a thermal insulator. The heat transfer is weakly dependent on $W e$ in this range of wall temperature. In the fingering film boiling regime $\left(T_{w 0}>450^{\circ} \mathrm{C}\right)$, the heat transfer decreases again with $T_{w 0}$. A clear separation of the curves for the different $W e$ can also be noticed above $T_{w 0}=500^{\circ} \mathrm{C}$. The variations of $Q_{w}$ are the result of the combined effects of several parameters: the local heat flux, the contact time and contact area, which evolve in a complex manner with $T_{w 0}$ which are discussed in [15].

\section{Conclusions}


The extremely rapid contact of a droplet which impinges on a superheated surface causes a very large superheating of the liquid. As long as the cooling of the surface occurring at the same time as the impact is sufficient, a wetting contact will take place. The dynamic LFP corresponds to the initial wall temperature for which the solid surface is cooled down to the temperature of the spinodal, i.e. the maximum temperature at which water can still exist in the liquid state. A model taking into account the heat transfer during the contact between the droplet and the wall, as well as the liquid flow in the spreading lamella, makes it possible to determine the dynamic LFP for sufficiently high Weber and Reynolds numbers. The Leidenfrost point is essentially a function of the thermal effusivity of the liquid and the wall. As the wall temperature approaches the LFP, the wetting area become narrows and the wetting lasts less and less time. Both parameters tending to 0 , become less and less dependent on the Weber number. The LFP is neither a minimum of the heat flux nor a minimum of the surface cooling. When the wall temperature exceeds the LFP, the liquid at the vapor interface remains in a very high level of superheating and in the absence of a wetting contact, the homogeneous nucleation can play an important role. It induces the formation of a fingering pattern and to a certain extent leads to a secondary atomization of tiny droplets if the drop spreading is sufficiently large.

\section{Acknowledgments}

The authors gratefully acknowledge the financial support of the CPER 2015-2020 ENERBATIN and of the European FEDER program.

\section{References}

[1] G. Liang, I. Mudawar, 2017, International Journal of Heat and Mass Transfer, 106, pp. 103126.

[2] J. D. Bernardin, I. Mudawar, 1999, Journal of Heat Transfer, 121 (4), pp. 894-903.

[3] C. Cai, I. Mudawar, H. Liu, C. Si, 2020, International Journal of Heat and Mass Transfer, 146, 118802.

[4] W. Wagner, A. Pruss, 2002, Journal of Physical and Chemical Reference Data, 31 (2), pp. 387-535.

[5] V. Carey, 1992, "Liquid-Vapor Phase-Change Phenomena: An Introduction To The Thermophysics Of vaporization and condensation in heat transfer equipment: An Introduction to the Thermophysics of Vaporization \& Condensation in Heat Transfer Equipment."

[6] F. M. Tenzer, J. Hofmann, I. V. Roisman, C. Tropea, Leidenfrost temperature in sprays: role of the substrate and liquid properties (2020). arXiv:2001.05426.

[7] M. Khavari, C. Sun, D. Lohse, T. Tran, 2015, Soft Matter, 11, pp. 3298-3303.

[8] W. Chaze, O. Caballina, G. Castanet, J.-F. Pierson, F. Lemoine, D. Maillet, 2019, International Journal of Heat and Mass Transfer, 128, pp. 469-478.

[9] D. Maillet, S. André, J.-C. Batsale, A. Degiovanni, C. Moyne, 2000, "Thermal quadrupoles: solving the heat equation through integral transforms."

[10] T. Tran, H. J. J. Staat, A. Prosperetti, C. Sun, D. Lohse, 2012, Phys. Rev. Lett., 108, 036101.

[11] J. Breitenbach, I. V. Roisman, C. Tropea, 2017, International Journal of Heat and Mass Transfer, 110, pp. 34-42.

[12] G. Castanet, W. Chaze, O. Caballina, R. Collignon, F. Lemoine, 2018, Physics of Fluids, 30 (12), 122109.

[13] J. M. Kolinski, S. M. Rubinstein, S. Mandre, M. P. Brenner, D. A. Weitz, L. Mahadevan, 2012, Phys. Rev. Lett., 108, 074503.

[14] I. V. Roisman, E. Berberovi, C. Tropea, 2009, Physics of Fluids, 21 (5), 052103.

[15] G. Castanet, O. Caballina, W. Chaze, R. Collignon, F. Lemoine, 2020, International Journal of Heat and Mass Transfer, 160, 120126. 